\title{
A Microbial Strategy to Multiply in Macrophages: The Pregnant Pause
}

\author{
Michele S. Swanson* and \\ Esteban Fernandez-Moreia
}

Department of Microbiology and Immunology, University of Michigan Medical School, Ann Arbor, MI 48109-0620. USA

* Corresponding author: Michele S. Swanson, mswanson@umich.edu

Humans live in harmony with much of the microbial world, thanks to a sophisticated immune system. As the first line of defense, macrophages engulf, digest, and display foreign material, then recruit specialists to eliminate potential threats. Yet infiltrators exist: certain fungi, viruses, parasites, and bacteria thrive within sentinel macrophages. By scrutinizing the life styles of these shrewd microbes, we can deduce how macrophages routinely mount an effective immune response. The bimorphic life cycles of three pathogens have dramatic consequences for phagosome traffic. In the transmissible state, Leishmania spp., Coxiella burnetii, and Legionella pneumophila block phagosome maturation; after a pregnant pause, replicative forms emerge and thrive in lysosomes.

Key words: autophagy, Coxiella, Legionella, Leishmania, lysosomes, macrophages, morphogenesis, phagosomes

Received 21 December 2001, revised and accepted for publication 4 January 2002

\section{Pathogens as Tools for Macrophage Biologists}

Pathogens employ a variety of strategies to survive in macrophages (1) (Figure 1). Toxoplasma gondii completely avoids fusion with the endosomal pathway; during invasion of mammalian cells, parasites direct the assembly of porous vacuoles that exclude host transmembrane proteins, presumably rendering their compartments invisible to the fusion machinery. In contrast, Mycobacterium tuberculosis stalls maturation of its phagosome and replicates within a recycling, early endosomal compartment. Even more bold are Leishmania spp., Coxiella burnetii, and Legionella pneumophila: These pathogens flourish in acidic lysosomal vacuoles, although not one arrives directly, or stays permanently.
To succeed, Leishmania spp., C. burnetii, and L. pneumophila employ biphasic life cycles. Cellular differentiation likely evolved in response to two selective pressures: the need to multiply and to be transmitted to a new host. To meet this dual challenge, all three pathogens alternate between an intracellular replicative state and a transmissible form that can tolerate harsh environments. In macrophages, the parasites' developmental cycle has dramatic consequences for phagosome biogenesis (Figure 1B). In the transmissible state, Leishmania spp., C. burnetii, and L. pneumophila block phagosome maturation; after a pregnant pause, replicative forms emerge that thrive in phagolysosomes.

Despite their distinct ecological habitats, Leishmania spp., $C$. burnetii, and L. pneumophila apply a similar strategy to surmount the macrophage barrier in humans. Recent conceptual and technical advances by laboratories studying one of the three microbes suggest a comparative approach is opportune. By considering that convergent evolution made these diverse pathogens members of the same exclusive club, we may gain fresh insight into how macrophages govern phagosome maturation and degrade foreign matter. After describing briefly their natural reservoirs and associated diseases, we review the experimental evidence that $L$. pneumophila, C. burnetii, and Leishmania spp. alternately avoid and exploit lysosomes to multiply in macrophages, and speculate on virulence mechanisms that may be shared.

\section{Separate Habitats, Diverse Diseases}

Leishmania: Prevalent in South America, the Leishmania are trypanosomatid protozoans transmitted among mammals by an insect vector (2). When sand flies bite an infected animal, macrophages harboring the pathogen are ingested with the blood meal. Released parasites multiply in the insect midgut, and these can be delivered during subsequent feedings to a new mammalian host. When humans are bitten, some Leishmania species establish self-limiting cutaneous lesions, whereas others spread systemically and cause kala azar, a fatal visceral disease.

Coxiella burnetii: C. burnetii is a gram-negative bacterium that infects a variety of winged, scaled and hoofed animals and, more rarely, the people in their proximity (3). For example, when infected female sheep give birth, they shed a large burden of micro-organisms. Consequently, humans can inhale the obligate intracellular bacterium in aerosols of con- 
A

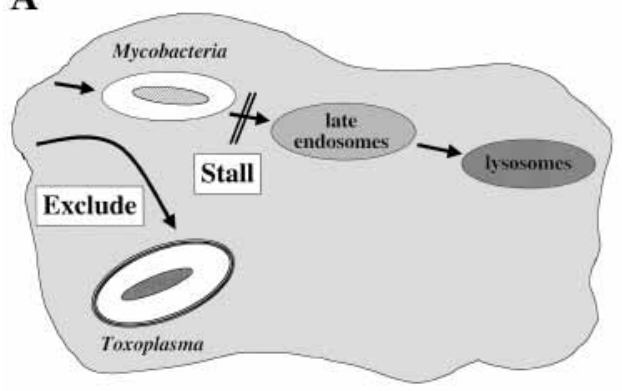

B

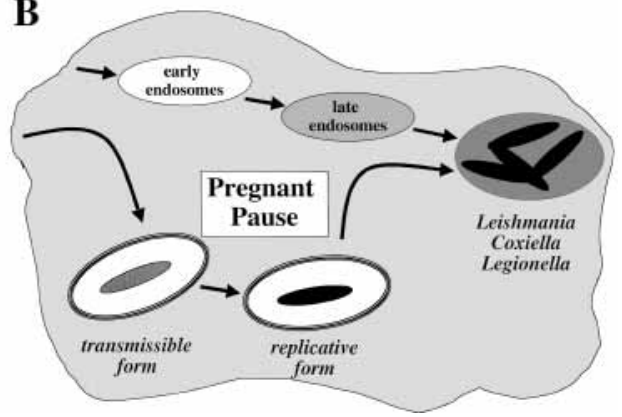

Figure 1: To avoid death in the lysosomes, pathogens short-circuit phagosome maturation. (A) Mycobacterium tuberculosis stalls maturation of its phagosome and replicates within an early endosomal compartment, whereas Toxoplasma gondii assembles a vacuole that excludes host transmembrane proteins. (B) The transmissible forms of Leishmania spp., Coxiella burnetii, and Legionella pneumophila establish vacuoles that are separate from the endosomal pathway, but later differentiate to replicative forms that thrive in acidic lysosomes.

taminated soil. When ingested by alveolar macrophages, the microbe can proliferate and disseminate, causing an acute flu-like syndrome known as $\mathrm{Q}$ fever. For an unlucky few, the infection progresses to chronic endocarditis or hepatitis.

Legionella pneumophila: Close cousins of $C$. burnetii, the legionellae are common in soil and water, where they thrive within amoebae (4). Humans typically become infected with L. pneumophila by inhaling contaminated aerosols generated by man-made devices, such as whirlpools or institutional air conditioners. This facultative intracellular pathogen can colonize alveolar macrophages and, if local or systemic host defenses are impaired, establish a life-threatening pneumonia. However, microbes are not transmitted from one person to another. Since humans are an evolutionary dead-end, it is apparent that pathogenic L. pneumophila emerged from communities of fresh-water amoebae (4). In fact, amoebae may be a natural reservoir for a variety of macrophage pathogens, including C. burnetii, M. avium, Burkholderia, Listeria. and Franciscella (5-7).

\section{Dimorphic Life Cycles}

Leishmania: To alternate between insects and mammals, parasites differentiate. Within the midgut of sand flies, Leishmania replicate as promastigotes, cells that are long, slender, flagellated, and covered by a dense filamentous glycocalyx. The predominant component of its coat is lipophosphoglycan (LPG), a glycosylphosphatidylinositol-linked polymer of phosphorylated disaccharide repeats. Among other traits, LPG mediates adherence to the midgut wall, protects the parasite from digestive enzymes and, as discussed below, blocks phagosome maturation $(8,9)$. When ingested by macrophages at the site of an insect bite, the protozoa differentiate to the amastigote form, which lack flagella and LPG, and replicate within acidic phagolysosomes. Upon return to the insect midgut during a subsequent blood meal, amastigotes that are released from macrophages differentiate to the promastigote form, and the cycle continues. In broth cultures, promastigotes become highly infectious in stationary phase
(10), but the signal transduction pathway that controls differentiation in vivo is not known.

Coxiella burnetii: C. burnetii also alternates between two morphologically distinct cell types $(11,12)$. The spore-like form, known as the small cell variant (SCV), tolerates extreme environments, whereas the large cell variant (LCV) replicates within eukaryotic cells. The architecture of LCVs is typical of gram-negative bacilli; in contrast, SCVs are roughly half their size, and their contents appear condensed. More recent molecular approaches confirmed that SCVs and LCVs are specialized cell types. Abundant in SCVs are a histone $\mathrm{H} 1$ homolog and ScvA, a 30-amino-acid basic peptide that localizes to condensed chromatin $(13,14)$. Conversely, homologs of the sigma factor RpoS and the translation factor EF-Tu are predominantly expressed by LCVs $(15,16)$. As described below, an acidic $\mathrm{pH}$ is required for SCV differentiation to the LCV form, but how the reciprocal event is regulated is not known. To a first approximation, the SCV state differs from the stationary phase of Escherichia coli, judging from its low quantity of the stationary phase sigma factor, RpoS (16).

Legionella pneumophila: The bimorphic life cycle of $L$. pneumophila reflects the selective pressures to replicate within and be transmitted among fresh-water amoebae. Growth conditions affect the physiology of this facultative intracellular bacterium $(4,17)$. Unlike bacteria cultured in broth, $L$. pneumophila released from macrophages or amoebae are short, wide, motile rods with a smooth, thick cell wall and abundant $\beta$-hydroxybutyrate. When compared to broth cultures, intracellular bacteria express a different profile of genes, proteins, membrane fatty acids, and lipopolysaccharide. Growth in amoebae also increases $L$. pneumophila resistance to biocides and antibiotics, invasion of mammalian cells, and virulence in mice (18-21). Thus, cellular differentiation enables L. pneumophila to alternate between extracellular and intracellular environments.

Recently, a regulatory model for $L$. pneumophila morphogenesis has been generated by genetic and molecular studies of 


\section{Swanson and Fernandez-Moreia}

broth and macrophage cultures. When amino acids become scarce, L. pneumophila accumulates the second messenger (p)ppGpp (22). As a result, the two-component regulator LetA/LetS and the sigma factors RpoS and FliA coordinate cellular differentiation to a transmissible form $(23,24)$. Accordingly, when conditions deteriorate, intracellular bacteria stop replicating and instead express a pore-forming cytotoxin $(25,26)$ to escape the host, osmotic resistance to tolerate environmental stress, motility to disperse, and factors that block phagosome maturation to establish a protected niche within the next phagocyte (27). When intracellular bacteria sense conditions favorable for growth, they down-regulate traits that promote transmission and convert to the replicative form.

\section{The 'Pregnant Pause' Strategy for Survival}

For all three pathogens, morphogenesis from an extracellular infectious form to an intracellular replicative phenotype occurs within a protected vacuole in macrophages. After a period of one to several hours, a new cell type emerges that is equipped not merely to tolerate but to multiply within the harsh lysosomal compartment. Thus, as detailed below, macrophage delivery of these pathogens to lysosomes benefits its intruder, not its host.

Leishmania: In macrophages, the infectious forms of Leishmania spp. establish vacuoles that are poorly fusogenic (Table 1). For example, by $2 \mathrm{~h}$ after formation, nearly $100 \%$ of phagosomes containing latex beads acquire BSA-gold from the endocytic pathway, but only $\sim 50 \%$ of those harboring promastigotes do so (28). A series of genetic and biochemical studies have demonstrated that the capacity of certain Leishmania spp. to block phagosome maturation is conferred by LPG, its unique surface glycoconjugate $(9,28,29)$. Mutants that lack LPG are readily delivered to lysosomes and are killed by macrophages (29-31). In particular, during the first hour of infection of $\mathbf{J 7 7 4}$ macrophages, almost $100 \%$ of the phagosomes formed by lpg2 mutant Leishmania donovani promastigotes accumulate Rab7 or LAMP-1, whereas only $\sim 50 \%$ of the vacuoles bearing wild-type promastigotes acquire these late endosomal features (30). In a reciprocal experiment, Holm and colleagues found that 30 min after ingestion by $\mathrm{J} 774$ macrophages, yeasts colocalize with LAMP-1, but yeasts opsonized with LPG do not (32).

The composition and production of LPG is developmentally regulated by Leishmania, and its pattern determines phagosome maturation (9). When cultured in broth, Leishmania major promastigotes become highly infectious in the stationary phase, concomitant with an increase in the average number of LPG repeating units and modifications of the side chain termini (10). In macrophages, when promastigotes differentiate to amastigotes, LPG expression is down-regulated. As a result, the parasites traffic to lysosomes, their replication niche (33) (Table 1). For example, 5 days after infection of mouse macrophages, Leishmania mexicana vacuoles contain LAMP- $1, \beta$-glucuronidase, and cathepsin D, and they are accessible to endocytic probes (34). Similarly, 2 days after

Table 1: Colocalization of young and mature pathogen vacuoles with markers of the endosomal pathway

\begin{tabular}{|c|c|c|c|c|c|c|c|}
\hline \multirow[t]{2}{*}{ Assay } & \multicolumn{2}{|c|}{$\begin{array}{l}\text { Coxiella } \\
\text { burnetii }\end{array}$} & \multicolumn{2}{|l|}{$\begin{array}{l}\text { Legionella } \\
\text { pneumophila }\end{array}$} & \multicolumn{2}{|c|}{$\begin{array}{l}\text { Leishmania spp } \\
\text { Promastigote }\end{array}$} & \multirow[t]{2}{*}{ Amastigote } \\
\hline & $2 \mathrm{~h}$ & $>6 h$ & $<8 \mathrm{~h}$ & $>8 \mathrm{~h}$ & $<3 d$ & $>3 d$ & \\
\hline \multicolumn{8}{|l|}{ Fluid phase markers } \\
\hline Lucifer Yellow & & $+(43)$ & & & & & \\
\hline Texas Red-ovalbumin & & & $-(49,51)$ & $+(51)$ & & & \\
\hline FITC-dextran & & $+(43)$ & & $+(51)$ & & $+(34)$ & \\
\hline BSA conjugates & & & & & $\operatorname{low}(28,30)$ & $+(34)$ & \\
\hline Electron dense colloids & $-(38)$ & $+(38,41)$ & $-(47,48,76)$ & & & $+(33)$ & \\
\hline \multicolumn{8}{|l|}{ Endosomal proteins } \\
\hline 5' nucleotidase & & $+(42)$ & $-(77)$ & & & & \\
\hline Transferrin receptor & & & $-(47,49)$ & & & & \\
\hline LAMP-1 & & $+(43)$ & $-(49,74,77)$ & $+(51)$ & $\operatorname{low}(30)$ & $+(34,35)$ & \\
\hline LAMP-2 & & $+(43)$ & & & & $+(34)$ & \\
\hline Proton ATPase & & $+(43)$ & & & & & \\
\hline Cathepsin D & & $+(43)$ & $-(49)$ & $+(51)$ & & $+(34,35)$ & \\
\hline Acid phosphatase & $-(38)$ & $+(38,41)$ & $-(48)$ & & & & \\
\hline rab7 & & & $\operatorname{low}(50,74)$ & & low (30) & $+(35)$ & \\
\hline \multicolumn{8}{|l|}{$\mathrm{pH}$} \\
\hline Acridine orange & & $+(43)$ & & & & & \\
\hline DAMP & & & & & & $+(36)$ & \\
\hline FITC & & $\sim 5.0(41,44)$ & $\sim 7.4(51,52)$ & $\sim 5.5(51)$ & & $\sim 5.0(36)$ & \\
\hline \multicolumn{8}{|l|}{ Inhibition of growth } \\
\hline Bafilomycin A & & $+(43)$ & & $+(51)$ & & & \\
\hline Lysosomotropic amines & & $+(43,45)$ & & $+(51)$ & & & \\
\hline
\end{tabular}


entering macrophages, L. donovani amastigotes colocalize with rab7, LAMP-1, and cathepsins $B, D, H$, and $L$ (35). As expected for lysosomal compartments, vacuoles harboring Leishmania amazonensis amastigotes are pH5.0 (36). The ability to culture amastigotes in broth will expedite analysis of the mechanisms that equip parasites to replicate in lysosomes (37).

Coxiella burnetii: Similar to the Leishmania, the infectious form of $C$. burnetii perturbs phagosome maturation (38). Exploiting ScvA as a morphogenesis marker (14), Howe and Mallavia monitored in parallel both the fate and the cellular differentiation of bacteria immediately after infection. Fifteen minutes after uptake by $\mathbf{J 7 7 4}$ macrophages, $\sim 70 \%$ of the intracellular bacteria remained in the SCV form, a fraction equal to that of the inoculum. Unlike killed bacteria, the majority remained separate from the lysosomal compartment, as judged by colocalization with acid phosphatase or endocytosed thorium dioxide. By $1 \mathrm{~h}, \sim 80 \%$ of the $C$. burnetii had differentiated to the metabolically active LCV form. Meanwhile, from 1 to $6 \mathrm{~h}$ postinfection, the fraction of Iysosomal bacteria increased from $\sim 30 \%$ to $\sim 60 \%$. Differentiation to the replicative form likely occurs prior to delivery to lysosomes, as SCV activation in vitro is more efficient at pH5.5 than at $\mathrm{pH} 7.0$ or 4.5 (38). Although correlative, the data are consistent with a model in which SCVs produce factors to block phagosome-lysosome fusion.

Whether the capacity to perturb phagosome maturation in macrophages is unique to the SCV form has not been tested rigorously. In fact, in a chicken egg model, SCVs and LCVs are equally infectious (11), but how accurately growth in eggs predicts a capacity to survive and replicate either in macrophages or an immunocompetent host is not clear. Another indication that LCVs may be infectious is that $2 \mathrm{~h}$ after Vero cells are infected with a mixed population of $C$. burnetii, intact LCVs are found in phagosomes that also contain SCVs (39). However, since wild-type L. pneumophila can chaperone avirulent trafficking mutants to a replication niche (40), this experiment does not establish that LCVs can alter phagosome biogenesis. More detailed experiments can determine whether $C$. burnetii must delay phagosome maturation to establish a replication niche in macrophages, and whether this attribute is unique to SCVs.

After differentiation to the LCV form, C. burnetii thrives in lysosomes (Table 1) (41-43). One detailed immunofluorescence microscopic analysis of a variety of infected eukaryotic cell lines demonstrated that after $40 \mathrm{~h}$ of infection, $C$. burnetii is located in vacuoles that contain the vacuolar proton ATPase, the late endosomal and lysosomal proteins LAMP-1 and LAMP-2, and the lysosomal protease cathepsin $D$ (43). In addition, compartments harboring the bacteria are accessible to fluid-phase endocytosis probes, they accumulate the lysosomotropic dye Acridine Orange, and their lumens are $\sim \mathrm{pH} 4.5-5.0(41,43,44)$. Furthermore, neutralization of the endosomal compartment by treatment with the proton ATPase inhibitor bafilomycin A or the weak bases am- monium chloride, chloroquine, or methylamine inhibits $C$. burnetii growth $(43,45)$.

Legionella pneumophila: Building on the pioneering work of Horwitz, several investigators have confirmed that, for several hours after formation, phagosomes harboring $L$. pneumophila remain separate from the lysosomal compartment (Table 1). Lysosomes labeled by acid phosphatase cytochemistry or by endocytosis of electron-dense colloids do not fuse with L. pneumophila phagosomes aged less than $6 \mathrm{~h}$ (4648), and the late endosomal and lysosomal proteins Rab7, LAMP-1, LAMP-2, and cathepsin D are not typical of bacterial phagosomes younger than $3 \mathrm{~h}(47,49,50)$. Furthermore, young $L$. pneumophila vacuoles do not accumulate material from the early endosomal compartment, including transferrin receptors, the endocytic tracers Texas Red-ovalbumin and Alexa Fluor-streptavidin, or the lipid dye CM-Dil, probes that are readily detected in phagosomes containing polystyrene beads or avirulent bacteria $(47,49)$. Therefore, in macrophages, $L$. pneumophila establishes a vacuole that remains functionally separate from the endosomal network for at least $6 \mathrm{~h}$.

In contrast, during their $10-15 \mathrm{~h}$ replication period, L. pneumophila phagosomes acquire Iysosomal characteristics (Table 1) (51). By $18 \mathrm{~h}$ postinfection, $70 \%$ of the vacuoles contain LAMP-1 and 50\% accumulate quantities of cathepsin D and fluorescent endocytic probes that can be detected by fluorescence microscopy. Moreover, whereas nascent $L$. pneumophila phagosomes remain at a neutral $\mathrm{pH}$, by 16$20 \mathrm{~h}$ after infection, those vacuoles that are accessible to fluid phase tracers are also acidic $(51,52)$. Thus, as they mature, $L$. pneumophila replication vacuoles merge with the lysosomal compartment. Four experimental results indicate that the Iysosomal compartment promotes, rather than inhibits, $L$. pneumophila growth (51). Vacuoles that contain greater than five bacteria also contain LAMP-1; bacteria within endocytic vacuoles can respond to a metabolic inducer by expressing a reporter Green Fluorescent Protein; neutralization of the endosomal pathway with the proton ATPase inhibitor bafilomycin A1 arrests bacterial replication; and replicating $L$. pneumophila obtained from macrophages are acid tolerant. Together, the data indicate that, like Leishmania and $C$. burnetii, $L$. pneumophila differentiates to a replicative form that thrives within macrophage lysosomes.

\section{Autophagy as an Alternate Route to Lysosomes}

The immature phagosomes established by some pathogens may be delivered to the lysosomes by autophagy (Figure 2). In this cellular response to stress or developmental cues (53), eukaryotic cells engulf cytoplasmic material and organelles, including mitochondria, within folds of the endoplasmic reticulum, or other as yet unidentified intracellular membranes. Autophagosomes then acquire LAMP-1, the proton ATPase, and lysosomal hydrolases, and the vacuolar contents are de- 


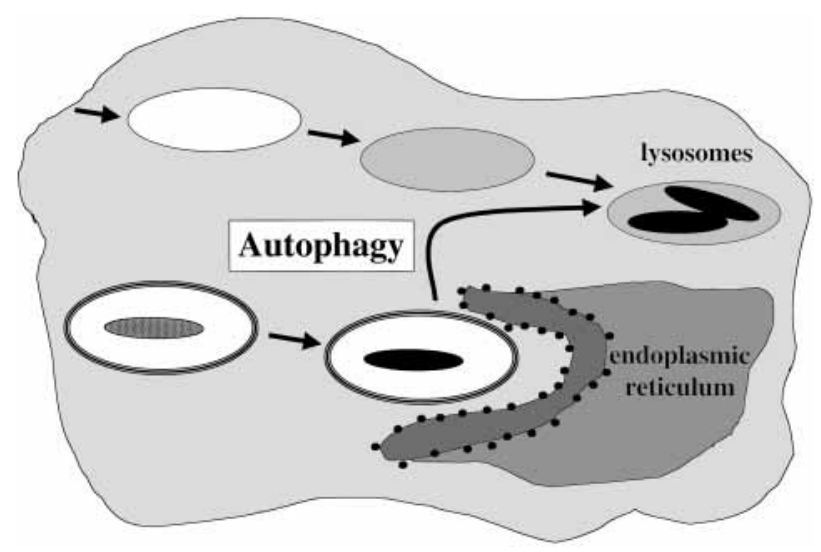

Figure 2: In macrophages, autophagy may act as a quality control mechanism that recognizes and redirects isolated pathogen vacuoles to the lysosomal compartment. See text for details.

graded. Several observations suggest that L. pneumophila traffic by autophagy to the lysosomal compartment: Endoplasmic reticulum encloses $L$. pneumophila vacuoles $4-6 \mathrm{~h}$ after formation $(54,55)$; Gsa7p (56), a ubiquitin-E1-like enzyme of the autophagy pathway, colocalizes with young $L$. pneumophila vacuoles (Amer and Swanson, unpublished observations); and conditions that stimulate autophagy promote association of the endoplasmic reticulum with the pathogen vacuole and also modestly stimulate bacterial growth (55). Finally, as expected for autophagosome maturation, the pathogen compartment slowly acquires lysosomal traits (51). Autophagy may also direct Leishmania to lysosomes, as vacuoles harboring amastigotes contain detectable calnexin, an endoplasmic reticulum protein, and they slowly accumulate cytosolic markers by a process sensitive to 3-methyladenine, an inhibitor of autophagy (57). Two other gram-negative pathogens, Brucella abortus and Porphyromonas gingivalis, reside within vacuoles that are enveloped by endoplasmic reticulum and acquire LAMP-1 $(58,59)$. Perhaps in macrophages, autophagy acts as a quality control mechanism for phagosome maturation. When pathogens assemble nonfusigenic vacuoles, the autophagy pathway may recognize the aborted phagosomes and redirect them to the lysosomal compartment.

\section{Adaptation to Lysosomes}

To replicate in the macrophage's most noxious organelle is an astonishing feat. Studies of $C$. burnetii metabolism revealed how pathogens can profit from the lysosomes' acidity. Transport and catabolism of glutamate by freshly explanted extracellular $C$. burnetii is optimal at $\mathrm{pH} 3-5$ and poor at neutral $\mathrm{pH}$ (60). These and other data indicate that intracellular C. burnetii exploit the proton gradient across its cytoplasmic membrane to drive critical biochemical reactions $(45,61,62)$. How lysosomal Leishmania, C. burnetii, and L. pneumophila cope with the array of degradative enzymes remains a mys- tery. The majority of cell biological studies demonstrating colocalization of pathogens with lysosomal proteases relied on immunological methods, techniques that do not address biological activity. However, infection by $C$. burnetii of $\mathrm{J} 774$ cells does not reduce total activity of lysosomal proteases (41). To investigate whether pathogens exploit the proteases of macrophage lysosomes to generate amino acids needed for replication requires more sophisticated probes of macrophage biology.

\section{Virulence factors that perturb phagosome maturation}

In the course of evolution, Leishmania, C. burnetii, and L. pneumophila have learned how to short-circuit phagosome maturation; therefore, they can direct us to critical regulators of this cellular pathway. One clue to how pathogens block phagosome maturation is the limited range of their virulence factors. Legionella pneumophila blocks its own progression to lysosomes with no apparent effect on phagosome acidification or maturation elsewhere in the phagocyte $(40,52)$. Likewise, when $\mathrm{J} 774$ macrophages are fed a mixture of latex beads and virulent Leishmania, 90\% of the beads still traffic to the endosomal compartment (28). Therefore, the virulence factors that block delivery of these pathogenic vacuoles to lysosomes act locally, most likely by altering either the phagosome's membrane or its membrane-associated regulatory factors.

Of the microbial factors that prevent phagosome maturation, the best understood is LPG. The current model for its mechanism of action incorporates the observed effects of purified LPG on target membranes (63). When L. donovani LPG partitions into bilayers, the membranes are stabilized and become refractory to fusion with enveloped viruses. Accordingly, LPG may inhibit phagosome maturation by increasing the energy required to alter the bilayer structure to form fusion intermediates. Intercalation of LPG into phagosomal membranes also inhibits protein kinase $\mathrm{C}$ activity and increases actin accumulation, two processes antagonistic to phagosome maturation $(32,64)$.

In light of LPG's potent inhibition of phagosome maturation, it is tempting to speculate that lipopolysaccharide serves an analogous function for the two gram-negative pathogens. By this model, developmental regulation of lipopolysaccharide would account for the pregnant pause of the phagosome maturation. Although lipopolysaccharide (LPS) variants of $C$. burnetii do occur $(65,66)$, they do not behave as elements of a developmental pathway. Phase 1 forms are isolated from patients or infected animals, have complex oligosaccharide side chains, are serum-resistant, and are infectious in a guinea pig model of disease. After passage in tissue culture or chicken eggs, Phase II organisms emerge; these cells express a less elaborate and more hydrophobic form of LPS (65), are serum-sensitive, adhere more readily to cultured macrophages, but are avirulent in guinea pigs (66). However, since the reciprocal conversion has not been reported, and both phases can establish persistent infections (67), there is no evidence that LPS modifications account for the trafficking pattern of $C$. burnetii in macrophages. 
Modifications of L. pneumophila lipopolysaccharide have also been correlated to virulence, but a link to growth phase or membrane trafficking has not been described. Legionella pneumophila produces an unusually hydrophobic lipopolysaccharide (LPS) that may facilitate its intracellular lifestyle (68). An LPS phase-variant mutant of $L$. pneumophila was isolated after repeat passage (69). Unlike wild-type, the mutant grew poorly within HL60 monocytic cells and in guinea-pig lungs. Furthermore, reversion of the mutant to the wild-type LPS was promoted during animal infections. Therefore, that a developmentally regulated phase variation of LPS contributes to L. pneumophila pathogenesis remains an attractive hypothesis.

The best characterized determinant of phagosome biogenesis of L. pneumophila is the Dot/lcm type IV secretion system. As discussed by Vogel in this issue (70), this specialized transport apparatus apparently acts during phagocytosis to establish the $L$. pneumophila replication vacuole. It is reasonable to predict that $C$. burnetii uses a similar strategy, as its genome encodes multiple homologs of the dot/icm genes. As yet, substrates of the Dot/lcm secretion complex that alter the fate of $L$. pneumophila or $C$. burnetii phagosomes have not been identified. Also contributing to L. pneumophila survival in macrophages is a Dot-independent activity (49). A surprising observation is that formalin-killed bacteria and viable dot mutant bacteria establish phagosomes that readily acquire LAMP-1, but do not accumulate cathepsin D, nor Texas Red-ovalbumin, CM-Dil, or Alexa Fluor-streptavidin, endocytic probes readily acquired by phagosomes containing E. coli or polystyrene beads. Therefore, in the transmissible form, L. pneumophila secretes a Dot/lcm-dependent factor and expresses a formalin-resistant surface factor to perturb phagosome maturation.

As observed for Leishmania, once L. pneumophila establishes a protected vacuole, virulence factors that block phagosome maturation are no longer needed. L. pneumophila that express DotA prior to contact with macrophages, but not after, still replicate during the primary infection cycle (50). and when $\operatorname{dot} A$ mutants share a phagosome with wild-type bacteria, they replicate (40). In addition, recent genetic studies of $L$. pneumophila virulence identified a two-component regulator, LetA/S, that induces transmission traits, including motility, cytotoxicity, and lysosome evasion, yet is dispensable for intracellular replication (24). Consistent with this genetic separation of the transmission and replication phenotypes, L. pneumophila that are replicating in macrophages down-regulate flagella production and cytotoxicity, and they traffic to the lysosomal compartment $(22,26,27,51)$. Therefore $L$. pneumophila transmission traits are dispensable for intracellular replication, a phenomenon analogous to the down-regulation of LPG by Leishmania spp. during differentiation from the transmissible to the replicative form.

\section{Odd Entrances May Point to Mechanism}

Coiling phagocytosis: Macrophages and amoebae engulf L. pneumophila within coils of plasma membrane $(71,72)$.
Coiling phagocytosis has been observed for a number of other microbes, including Leishmania donovani (73). Based on their detailed ultrastructural studies, Rittig and colleagues postulated that coiling phagosomes occur when the membranes of pseudopods that surround a particle fail to fuse (74). Accordingly, plasma membrane whorls may be a product of the same microbial activities that inhibit fusion with lysosomes, although this hypothesis has not been tested directly.

Perturbation of the actin cytoskeleton: Another striking aspect of pathogen entry that may provide insight to virulence mechanisms is aberrant actin dynamics. Isberg and colleagues have found that $L$. pneumophila can induce diffuse actin ruffles and formation of macropinosomes that contain GPI-linked proteins and also GM1-gangliosides, a hallmark of cholesterol-rich membranes (75). Similarly, C. burnetii induce dramatic and transient actin rearrangements in THP-1 monocytes (75), but their consequences for phagosome structure or function have not been determined. Finally, Leishmania LPG stimulates a prolonged accumulation of actin filaments around the phagosome, a trait that correlates to isolation from the endosomal pathway (32). How particular actin rearrangements affect pathogen fate is not known.

The formidable microbicidal activities of macrophages also function as strong selective pressures for variants that can replicate intracellularly. Leishmania spp., Coxiella burnetii, and Legionella pneumophila employ a 'pregnant pause' strategy to acquire the time needed to differentiate to a form that can exploit the harsh lysosomal compartment as a replicative niche. Knowledge of the tactics of macrophage pathogens provides a valuable conceptual framework for future research to identify and characterize virulence factors that perturb phagosome maturation. Because of their simplicity relative to mammalian cells, together with their more facile genetics, microbes promise to be powerful experimental tools to investigate macrophage cell biology.

\section{References}

1. Hackstadt T. Redirection of host vesicle trafficking pathways by intracellular parasites. Traffic 2000;1:93-99.

2. Herwaldt BL. Leishmaniasis. Lancet 1999;354:1191-1199

3. Norlander L. Q fever epidemiology and pathogenesis. Microbes Infect $2000 ; 2: 417-424$

4. Swanson MS, Hammer BK. Legionella pneumophila pathogenesis: a fateful journey from amoebae to macrophages. Annu Rev Microbiol 2000;54:567-613

5. La Scola B, Raoult D. Survival of Coxiella burnetii within free-living amoeba Acanthamoeba castellanii. Clin Microbiol Infect 2001;7:7579.

6. Cirillo JD, Falkow S, Tompkins LS, Bermudez LE. Interaction of Mycobacterium avium with environmental amoebae enhances virulence. Infect Immun 1997;65:3759-3767.

7. Winiecka-Krusnell J, Linder E. Free-living amoebae protecting Legionella in water: The tip of an iceberg? Scand J Infect Dis 1999;31:383-385 


\section{Swanson and Fernandez-Moreia}

8. Sacks DL, Modi G, Rowton E, Spath G, Epstein L, Turco SJ, Beverley SM. The role of phosphoglycans in Leishmania-sand fly interactions. Proc Natl Acad Sci USA 2000;97:406-411.

9. Turco SJ, Descoteaux A. The lipophosphoglycan of Leishmania parasites. Annu Rev Microbiol 1992;46:65-94

10. McConville MJ, Turco SJ, Ferguson MA, Sacks DL. Developmental modification of lipophosphoglycan during the differentiation of Leishmania major promastigotes to an infectious stage. EMBO J 1992;11:3593-3600.

11. Wiebe ME, Burton PR, Shankel DM. Isolation and characterization of two cell types of Coxiella burnetii phase I. J Bacteriol 1972;110:368377.

12. McCaul TF, Williams JC. Developmental cycle of Coxiella burnetii: structure and morphogenesis of vegetative and sporogenic differentiations. J Bacteriol 1981;147:1063-1076.

13. Heinzen RA, Hackstadt T. A developmental stage-specific histone $\mathrm{H} 1$ homolog of Coxiella burnetii. J Bacteriol 1996;178:5049-5052.

14. Heizen RA, Howe D, Mallavia LP, Rockey DD, Hackstadt T. Developmentally regulated synthesis of an unusually small, basic peptide by Coxiella burnetii. Mol Microbiol 1996;22:9-19.

15. Seshadri R, Hendrix LR, Samuel JE. Differential expression of translational elements by life cycle variants of Coxiella burnetii. Infect Immun 1999;67:6026-6033.

16. Seshadri R, Samuel JE. Characterization of a stress-induced alternate sigma factor, RpoS, of Coxiella burnetii and its expression during the development cycle. Infect Immun 2001;69:4874-4883.

17. Rowbotham TJ. Current views on the relationships between amoebae, Legionellae and man. Is J Med Sci 1986;22:678-689.

18. Barker J, Brown MRW, Collier PJ, Farrell ID, Gilbert P. Relationship between Legionella pneumophila and Acanthamoeba polyphaga: physiological status and susceptibility to chemical inactivation. Appl Environ Microbiol 1992;58:2420-2425.

19. Barker J, Scaife H, Brown MRW. Intraphagocytic growth induces an antibiotic-resistant phenotype of Legionella pneumophila. Antimicrob Agents Chemother 1995;39:2684-2688.

20. Cirillo JD, Falkow S, Tompkins LS. Growth of Legionella pneumophila in Acanthamoeba castellanii enhances invasion. Infect Immun 1994;62:3254-3261.

21. Brieland JK, Fantone JC, Remick DG, LeGendre M, McClain M, Engleberg NC. The role of Legionella pneumophila-infected Hartmannella vermiformis as an infectious particle in a murine model of Legionnaires' disease. Infect Immun 1997;65:5330-5333.

22. Hammer BK, Swanson MS. Co-ordination of Legionella pneumophila virulence with entry into stationary phase by ppGpp. Mol Microbiol 1999;33:721-731.

23. Bachman MA, Swanson MS. RpoS co-operates with other factors to induce Legionella pneumophila virulence in the stationary phase. Mol Microbiol $2001 ; 40: 1201-1214$.

24. Hammer BK, Suzuki E, Swanson M. A two-component regulator induces the transmission phenotype of stationary phase Legionella pneumophila. Mol Microbial 2002;in press.

25. Kirby JE, Vogel JP, Andrews HL, Isberg RR. Evidence of pore-forming ability by Legionella pneumophila. Mol Microbiol 1998;27:323-336.

26. Alli OAT, Gao L-Y, Pedersen LL, Zink S, Radulic M, Doric M, Abu-Kwaik $Y$. Temporal pore formation-mediated egress from macrophages and alveolar epithelial cells by Legionella pneumophila. Infect Immun 2000;68:6431-6440.

27. Byrne B, Swanson MS. Expression of Legionella pneumophila virulence traits in response to growth conditions. Infect Immun 1998;66:3029-3034.

28. Desjardins $M$, Descoteaux A. Inhibition of phagolysomal biogenesis by the Leishmania lipophosphoglycan. J Exp Med 1997;185:20612068.

29. Turco SJ, Spath GF, Beverley SM. Is lipophosphoglycan a virulence factor? A surprising diversity between Leishmania species. Trends Parasitol $2001 ; 17: 223-226$.

30. Scianimanico S, Desrosiers M, Dermine JF, Meresse S, Descoteaux A, Desjardins M. Impaired recruitment of the small GTPase rab7 correlates with the inhibition of phagosome maturation by Leishmania donovani promastigotes. Cell Microbiol 1999;1:19-32.

31. Spath GF, Epstein L, Leader B, Singer SM, Avila HA, Turco SJ, Beverly SM. Lipophosphoglycan is a virulence factor distinct from related glycoconjugates in the protozoan parasite Leishmania major. Proc Natl Acad Sci USA 2000;97:9258-9263.

32. Holm A, Tejle K, Magnusson KE, Descoteaux A, Rasmusson B. Leishmania donovani lipophosphoglycan causes periphagosomal actin accumulation: correlation with impaired translocation of PKC alpha and defective phagosome maturation. Cell Microbiol 2001;3:439-447.

33. Chang KP, Dwyer DM. Multiplication of a human parasite (Leishmania donovani) in phagolysosomes of hamster macrophages in vitro. Science 1976;193:678-680.

34. Russell DG, Xu S, Chakraborty P. Intracellular trafficking and the parasitophorous vacuole of Leishmania mexicana-infected macrophages. J Cell Sci 1992;103:1193-1210.

35. Lang T, Hellio R, Kaye PM, Antoine JC. Leishmania donovani-infected macrophages. characterization of the parasitophorous vacuole and potential role of this organelle in antigen presentation. J Cell Sci 1994; 107:2137-2150.

36. Antoine JC, Prina E, Jouanne C, Bongrand P. Parasitophorous vacuoles of Leishmania amazonensis-infected macrophages maintain an acidic pH. Infect Immun 1990;58:779-787.

37. Gupta N, Goyal N, Rastogi AK. In vitro cultivation and characterization of axenic amastigotes of Leishmania. Trends Parasitol $2001 ; 17: 150-153$.

38. Howe D, Mallavia LP. Coxiella burnetii exhibits morphological change and delays phagolysosomal fusion after internalization by J774A. 1 Cells Infect Immun 2000;68:3815-3821.

39. Heinzen RA. Intracellular development of Coxiella burnetii. In: Anderson B, Friedman H, Bendinelli M, eds. Rickettsial Infection and Immunity. New York: Plenum Press; 1997. p. 99-129.

40. Coers JN, Monahan C, Roy CR. Modulation of phagosome biogenesis by Legionella pneumophila creates an organelle permissive for intracellular growth. Nat Cell Biol 1999.

41. Akporiaye ET, Rowatt JD, Aragon AA, Baca OG. Lysosomal response of a murine macrophage-like cell line persistently infected with Coxie/la burnetii. Infect Immun 1983;40:1155-1162.

42. Burton PR, Stueckemann J, Welsh RM, Paretsky D. Some ultrastructural effects of persistent infections by the rickettsia Coxiella burnetii in mouse L cells and green monkey kidney (Vero) cells. Infect Immun 1978;21:556-566.

43. Heinzen RA, Scidmore MA, Rockey DD, Hackstadt T. Differential interaction with the endocytic and exocytic pathways distinguish parasitophorus vacuoles of Coxiella burnetii and Chlamydia trachomatis. Infect Immun 1996;64:796-809.

44. Maurin MA, Benoliel AM, Bongrand P, Raoult D. Phagolysosomes of Coxiella burnetii-infected cell lines maintain an acidic $\mathrm{pH}$ during persistent infection. Infect Immun 1992;60:5013-5016.

45. Hackstadt T, Williams JC. Biochemical stratagem for obligate parasitism of eukaryotic cells by Coxiella burnetii. Proc Natl Acad Sci USA $1981 ; 78: 3240-3244$.

46. Berger $\mathrm{KH}$, Isberg RR. Two distinct defects in intracellular growth complemented by a single genetic locus in Legionella pneumophila. Mol Microbiol 1993;7:7-19.

47. Clemens DL, Horwitz MA. Characterization of the Mycobacterium tuberculosis phagosome and evidence that phagosomal maturation is inhibited. J Exp Med 1995;181:257-270.

48. Horwitz MA. The Legionnaires' disease bacterium (Legionella pneumophila) inhibits phagosome lysosome fusion in human monocytes. J Exp Med 1983;158:2108-2126. 
49. Joshi AD, Sturgill-Koszycki S, Swanson MS. Evidence that Dot-dependent and -independent isolate the Legionella pneumophila phagosome from the endocytic network. Cell Microbiol 2001;3:99-114.

50. Roy CR, Berger KH, Isberg RR. Legionella pneumophila DotA protein is required for early phagosome trafficking decisions that occur within minutes of bacterial uptake. Mol Microbiol 1998;28:663-674.

51. Sturgill-Koszycki S, Swanson MS. Legionella pneumophila replication vacuoles mature into acidic, endocytic organelles. J Exp Med 2000;192:1261-1272.

52. Horwitz MA, Maxfield FR. Legionella pneumophila inhibits acidification of its phagosome in human monocytes. J Cell Biol 1984:99:1936-1943.

53. Kim J, Klionsky DJ. Autophagy, cytoplasm-to-vacuole targeting pathway, and pexophagy in yeast and mammalian cells. Annu Rev Biochem 2000;69:303-342.

54. Horwitz MA. Formation of a novel phagosome by the Legionnaires disease bacterium (Legionella pneumophila) in human monocytes. J Exp Med 1983;158:1319-1331.

55. Swanson MS, Isberg RR. Association of Legionella pneumophila with the macrophage endoplasmic reticulum. Infect Immun 1995: 63:3609-3620

56. Yuan W, Stromhaug PE, Dunn WA, Jr. Glucose-induced autophagy of peroxisomes in Pichia pastoris requires a unique E1-like protein. Mo Biol Cell 1999;10:1353-1366.

57. Schaible UE, Schlesinger PH, Steinberg TH, Mangel WF, Kobayashi T, Russell DG. Parasitophorous vacuoles of Leishmania mexicanao ac quire macromolecules from the host cell cytosol via two independent routes. J Cell Sci 1999;112:681-693.

58. Dorn BR, Dunn WA, Jr, Progulske-Fox A. Porphyromonas gingivalis traffics to autophagosomes in human coronary artery endothelial cells. Infect Immun 2001;69:5698-5708.

59. Pizarro-Cerda J, Meresse S, Parton RG, van der Goot G, Sola-Landa A, Lopez-Goni I, Moreno E, Gorvel JP. Brucella abortus transits through the autophagic pathway and replicates in the endoplasmic reticulum of nonprofessional phagocytes. Infect Immun 1998;66:5711-5724

60. Hackstadt T, Williams JC. pH dependence of the Coxiella burnetil glutamate transport system. J Bacteriol 1983;154:598-603.

61. Hackstadt T, Williams JC. Stability of the adenosine $5^{\prime}$-triphosphate pool in Coxiella burnetii: influence of $\mathrm{pH}$ and substrate. J Bacteriol $1981 ; 148: 419-425$

62. Hackstadt T. Estimation of the cytoplasmic pH of Coxiella burnetii and effect of substrate oxidation on proton motive force. J Bacterio 1983:154:591-597.

63. Miao L, Stafford A, Nir S, Turco SJ, Flanagan TD, Epand RM. Potent inhibition of viral fusion by the lipophosphoglycan of Leishmania donovani. Biochemistry 1995;34:4676-4683.
64. Giorgione JR, Turco SJ, Epand RM. Transbilayer inhibition of protein kinase $\mathrm{C}$ by the lipophosphoglycan from Leishmania donovani. Proc Natl Acad Sci USA 1996:93:11634-11639.

65. Ftacek P, Skultety L, Toman R. Phase variation of Coxiella burnetii strain Priscilla: influence of this phenomenon on biochemical features of its lipopolysaccharide. J Endotoxin Res 2000;6:369-376.

66. Moos A, Hackstadt T. Comparative virulence of intra- and interstrain lipopolysaccharide variants of Coxiella burnetii in the guinea pig model. Infect Immun 1987:55:1144-1150.

67. Baca OG, Akporiaye ET, Aragon AS, Martinez IL, Robles MV, Warner NL. Fate of phase I and phase II Coxiella burnetii in several macrophage-like tumor cell lines. Infect Immun 1981;33:258-266.

68. Knirel YA, Rietschel ET, Marre R, Zahringer U. The structure of the Ospecific chain of Legionella pneumophila serogroup 1 lipopolysaccharide. Eur J Biochem 1994;221:239-245.

69. Lüneberg $E$, Zähringer $U$, Knirel $Y A$, Steinmann $D$, Hartmann $M$, Steinmetz I, Rohde M, Kohl J, Frosch M. Phase-variable expression of lipopolysaccharide contributes to the virulence of Legionella pneumophila. J Exp Med 1998;188:49-60.

70. Sexton JA, Vogel JP. Utilization of type IVB secretion systems by intracellular pathogens. Traffic 2002:3:178-185.

71. Horwitz MA. Phagocytosis of the Legionnaires' disease bacterium (Legionella pneumophila) occurs by a novel mechanism: engulfment within a pseudopod coil. Cell 1984;36:27-33.

72. Bozue JA, Johnson W. Interaction of Legionella pneumophila with Acanthamoeba castellanii: uptake by coiling phagocytosis and inhibition of phagosome-lysosome fusion. Infect Immun 1996;64:668673

73. Rittig MG, Schroppel K, Seack K-H, Sander U, N'Diaye E-N, Maridonneau-Parini I, Solbach W, Bogdan C. Coiling phagocytosis of Trypanosomatids and fungal cells. Infect Immun 1998:66:4331-4339.

74. Rittig MG, Burmester G-R, Krause A. Coiling phagocytosis: when the zipper jams, the cup is deformed. Trends Microbiol 1998;6:384-388.

75. Watarai M, Derre I, Kirby J, Growney JD, Dietrich WF, Isberg RR. Legionella pneumophila is internalized by a macropinocytotic uptake pathway controlled by the Dot/lcm system and the mouse Lgn1 locus. J Exp Med 2001;194:1081-1096.

76. Meconi S, Jacomo V, Boquet P, Raoult D, Mege JL, Capo C. Coxiel/a burnetii induces reorganization of the actin cytoskeleton in human monocytes. Infect Immun 1998;66:5527-5533.

77. Berger $\mathrm{KH}$, Merriam JJ, Isberg RI. Altered intracellular targeting properties associated with mutations in the Legionella pneumophila dotA gene. Mol Microbiol 1994;14:809-822

78. Clemens DL, Horwitz MA. Membrane sorting during phagocytosis. selective exclusion of major histocompatibility complex molecules but not complement receptor CR3 during conventional and coiling phagocytosis. J Exp Med 1992;175:1317-1326. 